\title{
Automated Pay and Use Browsing and Printing Machine
}

\author{
P.Parameswari, S.Thangavel, E.B.Priyanka
}

\begin{abstract}
The existing system function as a multiple unit like computer setup and printing unit is fixed at different location and an intermediate person will be there to do the printing process. The existing system faces some difficulties like structure is not compact, all the units are installed at a different location, Customer needs $24 * 7$ service, but the service is not available in all the times. The service timing is limited up to the availability of the labor. The Automated pay and use browsing, and printing machine is a type of vending machine which performs $24 * 7$ browsing and printing service. The system can be implemented in the public places, educational institutions etc. The setup works on the time-based control system based on the amount paid as the input for browsing and printing separately. The main controller is the Raspberry Pi which controls and monitors the complete browsing and printing process. The separate coin collector device is interfaced with it to collect the amount for browsing and printing service. A continuous monitoring is done through IoT which helps to monitor the machine $24 * 7$ which provides data like number of printout taken, browsing time. This makes the performance monitoring process easy.
\end{abstract}

Keywords: Internet of Things, pay and use browsing machine.

\section{INTRODUCTION}

The today's world is running behind the technology of automation with upgradation of the latest advancement. It incorporates with reducing the human effort and time involved in the customer's side. In all areas of shopping malls and all relevant purchasing sectors, delivering the products based on the selection of particular products on their interest with the give up of inserting coins or paying cards. Now a day's vending machines plays vital role for best example of automation in the retail shops. The development of technological equipment is mainly for utilizing the importance of time resources of human life. The main engineering principle which get best suit to the automation is the Automatic Teller Machine (ATM). By following the most domestic application of engineering ideas such as chocolate vending machines, cool drink machines. In the application oriented area to implement the automation, Automatic pay and use browsing and printing machine design and features establishment is developed. This machine is automated for providing 24 hours service for the customers.

Ammar et al (2014) combined RFID technology with

Revised Manuscript Received on August 05, 2019.

P.Parameswari, Department of MCA, Kumaraguru College of Technology, Coimbatore, Tamilnadu, India.

S.Thangavel, Department of Mechatronics Engineering, Kongu Engineering College, Perundurai, India

(E-mail: thangavel.mts@kongu.ac.in)

E.B.Priyanka, Department of Mechatronics Engineering, Kongu Engineering College, Perundurai, India

(E-mail: priyankabhaskaran1993@gmail.com) wireless sensor network through which RFID reader senses the signal and communicates that information of the environment to the processor wirelessly for developing bust ticket vending machine. Mahdi et al (2014) Creates public transport ticketing system and implemented in the real-time area of Bangladesh for the people to reduce human concern problems and traffic jam due to the vehicles during peak hours. Ana et.al (2014) designed automatic finite state based billing machine with microprocessor as the controller to regulate the functioning of the programmed vending operation. Hence the objective is to design an automated browsing and printing machine and to fabricate the machine for providing $24 \times 7$ service for the customers by interfacing with IoT for providing continuous monitoring.

\subsection{Existing \& Proposed Browsing \& Printing System-}

\section{Demerits/Merits}

The existing system demands manual work and the components are not functioned as a single unit. Labor is required for maintaining the browsing time and cost for printing. Customer needs $24 \times 7$ service, but the service is not available in all the times and the service timing is limited up to the availability of the labor. But the merit of the proposed is that the implementation of automation to the whole system when the programmed coding accepts the defined transaction at the proper procedure. The portable feature is incorporated with this automated browsing and printing machine to the other area on the requirement of necessity. It starts to continue its functioning when it is given with the proper power supply and can be utilized whenever the person needs browsing through the $24 * 7$ basis.

\section{PROPOSE AUTOMATED PAY \& USE BROWSING AND PRINTING SYSTEM}

The design of an automated browsing and printing machine starts with preparation of 2D CAD model as shown in the below figure. The Mechanical structure of the machine model is fabricated using plywood and the components are placed in the mechanical setup. The entire process is controlled using a Raspberry pi controller. The programming is done using python software; a LCD monitor interface is used for browsing and a printer is used for printing. A coin acceptor is interfaced for providing the money based on the required output. The entire process can be monitored using IoT.

Internet of things plays vital role in the automation sector to communicate existing measuring area to the cloud platform through proper communication channel. The data 
is communicated from the measuring world to the virtual sector through application protocols. An IOT system consists of three main categorical areas such as real world involving objects to the cloud server through wireless communication channel to transfer data from the field area.Figure 1 gives the essential sequences involved in the IoT system in the structured manner.

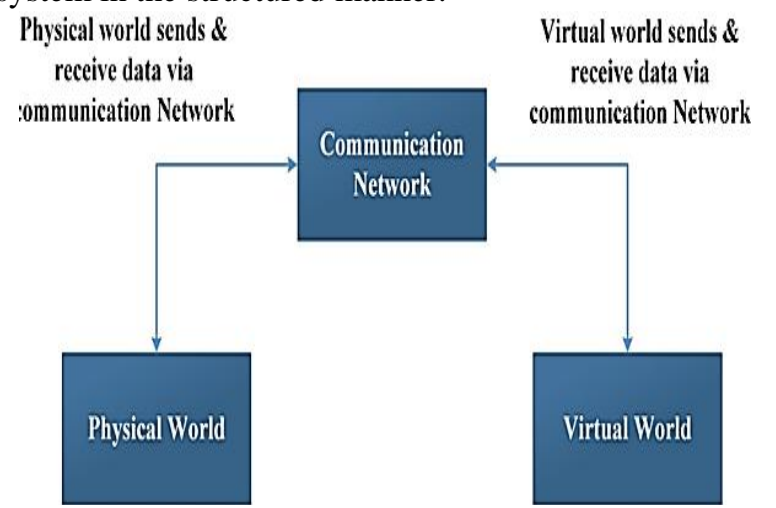

Figure 1. Block diagram representation of Internet of Things.

\section{EXECUTING METHODOLOGY OF AUTOMATED BROWSING \&PRINTING SYSTEM \& RESULTS}

The automated browsing and printing machine is provided with a coin acceptor, which accepts the coins of the customer and provides the service. The time for browsing is based on the amount provided by the customer. The printing process separately requires the customer to provide the coin. The response will be closed within the time provided. If the user wants to continue browsing, another coin is to be inserted. The user interface screen is user friendly. Sample coin is placed in the coinacceptor. It only accepts coins as that the of sample coin. If other coin is inserted, the coin will be rejected by it. The user interface scree will be available only after the coin is inserted into the coin acceptor. The entire process is controlled using a Raspberry pi controller. The programming is done using python software; a LCD monitor interface is used for browsing and a printer is used for printing the entire process can be monitored through IoT. It works with continuous monitoring of operation, hence within the given operating conditions, the chances of failure in the machine will be less. The fabrication involved in the proposed browsing and printing machine follows simple mechanical equipment involvement. The human effort is not all involved and its usage and maintenance decides the life span of the proposed system. The machine contains coin acceptor and an additionally bar code scanning which makes user friendly operation tothe customer. The mechanical and electrical components utilized are given in Table 1.

Table.1 Components implemented in automated browsing \& printing system

\begin{tabular}{|l|l|}
\hline Materials & Specifications \\
\hline Raspberry Pi & Pi3, model B ARMv8 64bit \\
\hline Coin acceptor & COM-11719 (3-coin type) \\
\hline Monitor & 14-inch, LCD monitor \\
\hline Printer & HP, desk jet color printer 2135 \\
\hline Plywood & $5 \mathrm{~mm}$ \\
\hline
\end{tabular}

The Automated pay and use browsing, and printing machine is a type of vending machine which performs $24 \mathrm{x} 7$ browsing and printing services. The main controller is the Raspberry $\mathrm{Pi}$ which controls and monitors the complete browsing and printing process. The corresponding work flow is given in figure 3 .

The power supply is fed to the input, output devices and the controller. All the components are interfaced with each other using the wire. At first, the proximity sensor feeds the input to the controller depending on which the controller produces the respective output signals to the output devices.

Raspberry Pi holding the Broadcom GH789.12 feature serves as the main controller with1.2GHz Quad-Core ARM Cortex-A12 processor in the main chip to recognize and sequence the received signals. For advanced data communication, it is supported with $802.11 \mathrm{~b} / \mathrm{g} / \mathrm{n}$ Wireless LAN and Bluetooth with proper data modem. The multiplexing signal as packet with the baud rate of 900ns will be transmitted with DMA filter with high frequency counter. The main CPU process the signal and segregate into finite interval coded packets with error detection.The coin acceptor will take the coin which is same as the sample coin provided. The main sensor in the proposed browsing and printing machine initially measures the size, weight and number image detection. Its programmed coding will be sub-grouped that will be taken based on the user selection. The user after inserting the coin, selects the button pointing the inserting coin count rate. Based on the analysis, if the coding gets correlated with the inserted coin, the seven segment display indicate the acceptance of coin and permit to proceed the browsing procedure. The monitor in the display holds tristate logic transistor with liquid crystal display with the indication of back lighting. When the inserted coin is mismatched, the user cannot be permitted to proceed the further attempt of using the fabricated automation machine. 


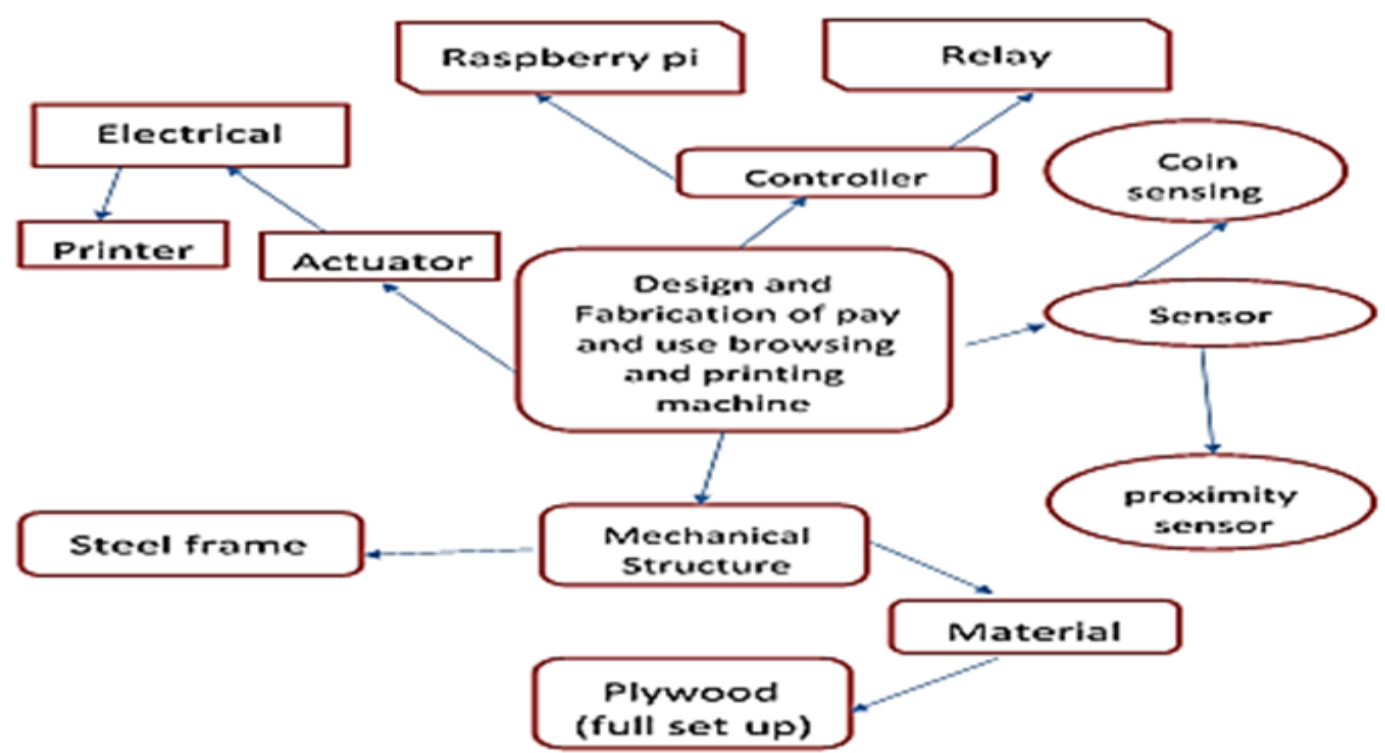

Figure 2. Conceptual design of proposed automated system

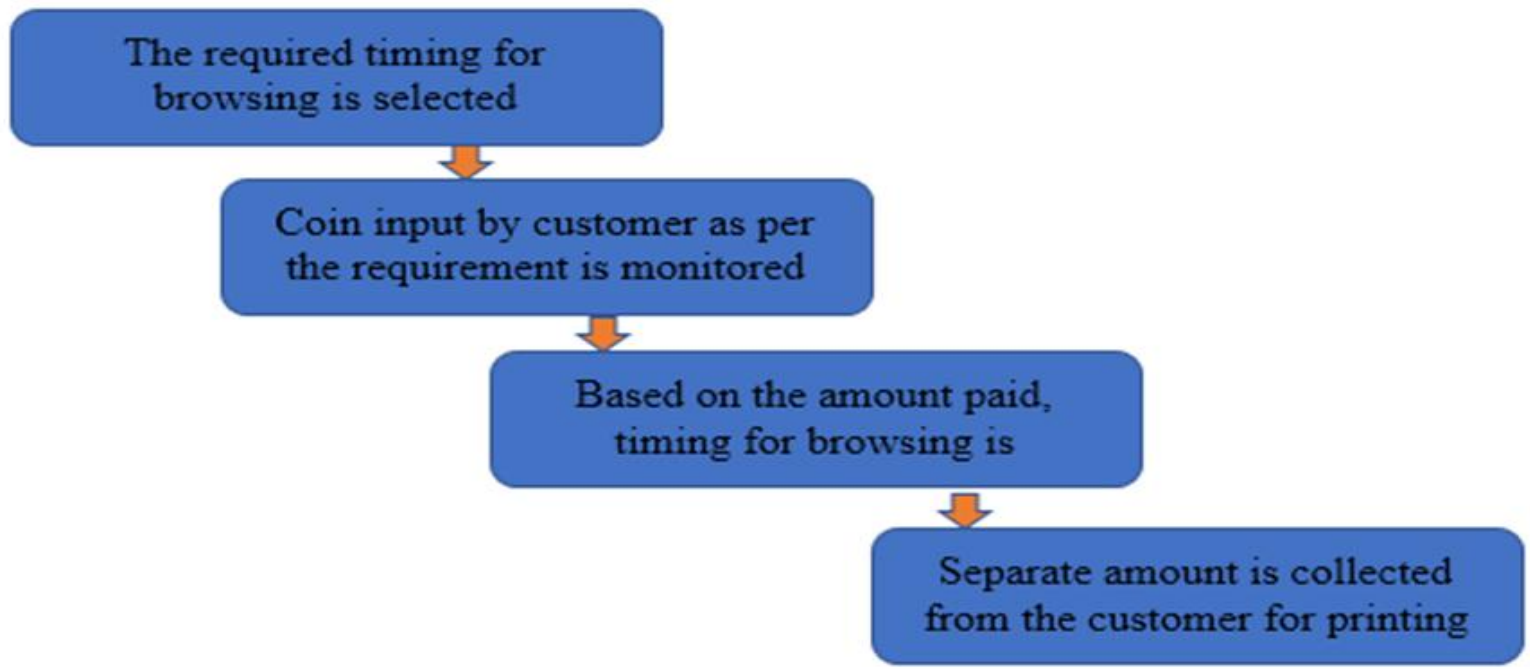

Figure 3.Block diagram of work flow in the automated process

The monitor resolution for the computer setting is given with the ratio rate of $4: 3$ to $16: 9$. In this project HP desk jet printer is used because Raspberry Pi can be interfaced only with these printer and to implement IoT.

In the developed machine, plywood is the material developed from the wood grain placed with the perpendicular section of one above the layer to form the sheet structures. The electronics circuit section consists of proximity sensor, contactor and relay to perform programmed control functions. The proximity sensor is used to detect the insertion of coin to detect the physical presence. The sensor generates the electromagnetic field with the beside objects, when the user insert the coin, the radiation field gets changed. The corresponding current signal passes to the relay circuit powered with the main power supply. It prevents the excessive voltage or current from the proximity sensor and power supply, hence the relay used to provide switching operations. The electric motor is actuated by receiving the current signal through contactor from the relay control unit. The programmed embedded system is synchronized with the IoT application to execute the further sets to the user to proceed with browsing process is shown in Figure 4.

Processing and Feedback Devices-The input sensor and output actuator are inter related with each other to exchange information between the system sensors utilized. The obtained real insight physical quantities data are transmitted to the cloud server through proper communication networks. The processing input sensor receives data parallel processed with the controller to actuate the electric motor as output channel. Through data oriented network band the information is passed to the IoT cloud platform. The application layer sends back the useful feedback signal from the cloud to the real world printing machine.

Gateway Requirements:The IoT application is oriented with the gateway device for proper data communication. The data is transferred with the transmission control and internet protocol since the feedback is accompanied with internet utility services. The real-time data and handshake signal from the cloud server are interfaced with the gateway

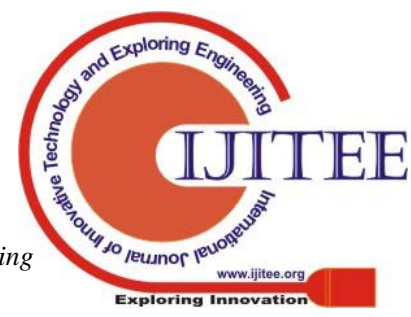


device. The Linux operating system is installed as Java platform to work on the execution of Windows 10 OS. The Zigbee interface is followed with binary digit coding structure with computing technique to transfer data. IPV6 address routing scheme is passed to receive token for final confirmation data from the cloud.

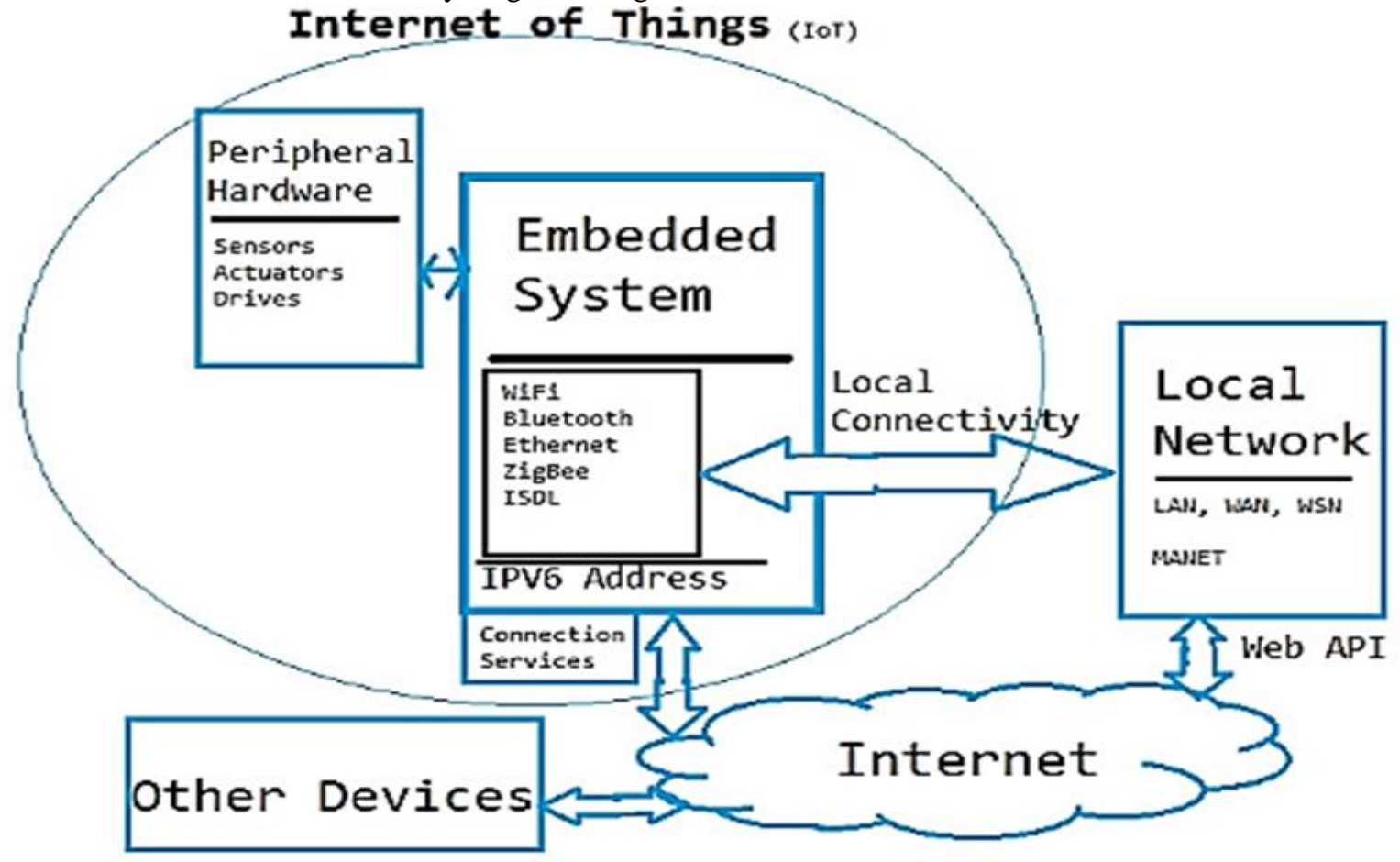

Figure 4. Proposed system with IoT architecture
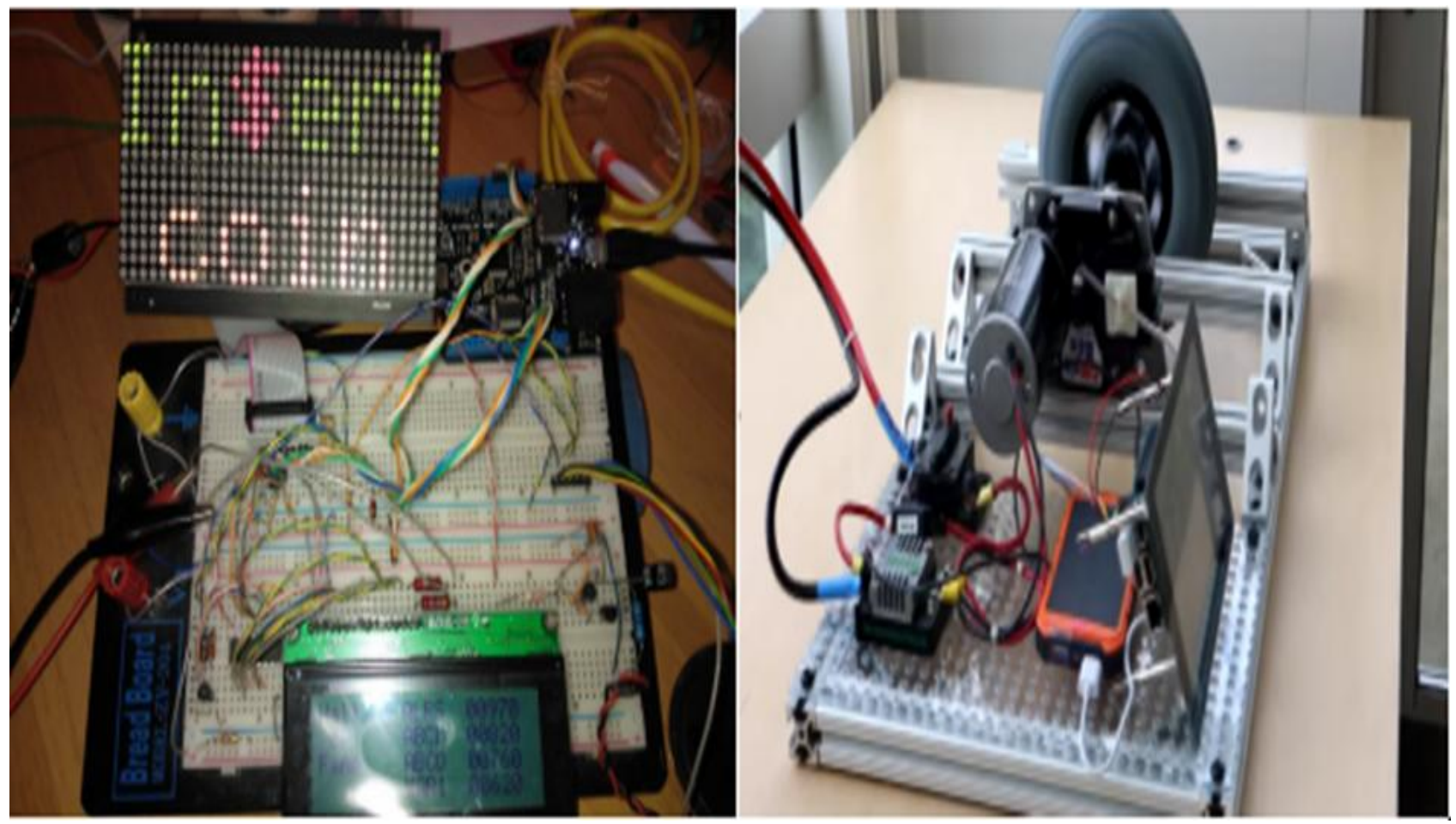

Figure 5. Configuration of coin setting and interfacing with printing motor from PC.

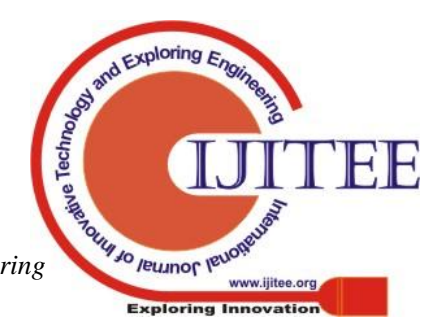


.For knowledge to vanish on the realization of the manipulator, the Internet of Things hassles: (1) a collectivesympathetic about the circumstances of utilizing handlers and industrialized smart sensors,(2) structural design of software and unescapable data transmissionpathway to tackle and transfer the most pertinent circumstantial associated data and (3) self-directed and adaptive performance by its analysis algorithm tool incorporated with IoT.

By unifying basic three essential substructure leads to smooth-reliable connectivity by real-time data manipulation from the implemented industrial sector of a complex environment.To overcome the limitations of conventional utility systems, the proposed design of the IoT framework offers new utility monitoring and control system can flexibly accommodate changes made to improve the operational efficiency of utilities.

Data propagation- The data propagated as based on the routing protocol with loss network with master-slave configuration. The network link layer of key segment allocation of bandwidth with both low and high bands of 450-1200 MHz frequency. The guard rate varies with respect to the signal strength of gateway.

Cloud priority - Internet of Things (IoT) inferred as theidentified Internet of Objects becomes an emergent technology that is widely used by several application domains. Thanks to its advanced services, the International Telecommunication Union (ITU) outlines to the real world in the Information Society as powerful infrastructure, empowering innovative options on interrelatingexisting and evolving platforms, interoperable statistics and communication expertise. Diverse fields of applications adapt IoT in their main implementation process. The most protruding areas of solicitation taken in the smart home, smart energy, healthcare, manufacturing, transport, environment, smart industry and so on.

IOT Application - The data analysis is done with IaaS cloud structure based on the infrastructure as a service. The virtual private cloud takes control and storage of browsing and printing data details by giving actuated feedback signal to the mechanical actuator of the system. The complete automation for browsing and printing with IoT embedded system is designed supported from the cloud to operate printer and computer for browsing and keep records of sufficient system.

\section{CONCLUSION \& FUTURE SCOPE}

Thus, automatic system for browsing and printing is fabricated and experimentally validated in real-time by interfacing all the mechanical, electrical and electronics systemto provide $24 \times 7$ service for the customers using IoT. The machine opens the user interface screen, after inserting the coin and the response will be closed within the provided time. Number of print taken is send to the corresponding mobile phone. The future scope is to make the availability of this automated machine in all public places, educational institutions and offices.

\section{CONFLICT OF INTEREST}

All the contributors in this research work have no clashes of attention to announce and broadcasting this article.

\section{ACKNOWLEDGMENT}

This research work is carried out under the Senior Research fellowship received from CSIR(Council for Scientific and Industrial Research) with grant no.678/08(0001)2k18 EMR-I.

\section{REFERENCES}

1. Ameer H. Morad(2010)," GPS Talking for Blind People", Journal of emerging technologies in web intelligence, Vol. 2, No. 3.

2. Ben Ammar Hatem Hamam Habib(2009)," Bus Management System Using RFID In WSN",European and Mediterranean Conference on InformationSystems 2010(EMCIS2010), Abu Dhabi, UAE.

3. FoisalMahediHasan(2010), Golam Tangim, Md. Kafiul Islam, Md. RezwanulHaqueKhandokar,ArifUlAlam," RFID-based Ticketing for Public Transport System: Perspective MegacityDhaka".

4. R. S. Khurmi\& J. K. Gupta(1998), Machine Design, Manufacturing considerations First Edition.

5. Nagaraj Balakrishnan, Reshmi S., and R. Arunkumar. "Smart real time rescue system for fishermen." Pak J Biotechnol 15, no. 1 (2018): 73-75.

6. ROBERT A. SOHLEGEL(2002), Google Scholar, Vending Machine.

7. William Bolton(1995), Mechatronics a Multi-disciplinary approach, Fourth edition.

8. William Bolton(1999), Mechatronics, a Multidisciplinary approach, DC motor control, pp 201-208.

9. William Bolton(1999), Mechatronics, a Multidisciplinary approach, Micro controller \& ADC control, pp 339-465.

10. Pugazhendhiran(2010), Electric motors and Drives Control., EDC sixth edition. 